\title{
Habitus, Hierarchien und Methoden: „Feine Unterschiede" zwischen Physik und Chemie
}

\author{
Carsten Reinhardt
}

\begin{abstract}
Habitus, Hierarchies, and Methods: Subtle Distinctions between Physics and Chemistry
Research methods and their developers play a crucial role in bringing together scientific fields. Scientists, who wish to have their methods acknowledged and used in another discipline have to bridge the gaps between different practices and worldviews, and they often experience hostility, incredulity, and in general feelings of ,otherness', while changing the established practice in a scientific field. Researchers representing newly emerging fields such as materials science have to overcome obstacles that are caused more by feelings of threat and fear than by arguments rooted in epistemic or social considerations. In using the habitus concept of Pierre Bourdieu, I analyze in this article statements and observations of the scientists who in the 1950s and 1960s have introduced physical methods into chemistry and in so doing merged characteristic features of research communities into a new unit. The habitus of physicists, chemists and method-oriented researchers are shaped by hierarchical relationships inside the disciplines, and between them. Studying emotional expressions in interviews and self-descriptions gives access to hitherto neglected parts of the historical processes leading to new disciplines and fields. The brief case studies presented are seen as exemplars, and the analysis offered is intended more to provide a possible model for further research than as giving conclusive answers.
\end{abstract}

Keywords: Bourdieu, physical methods, interdisciplinary fields, habitus, hierarchy

Schlüsse/wörter: Bourdieu, Physikalische Methoden, Interdisziplinäre Felder, Habitus, Hierarchie

Als der US-amerikanische Chemiker Herbert S. Gutowsky im Jahr 1950 dem theoretischen Physiker Norman Ramsey ein Experiment vorschlug, um die Theorie der kernmagnetischen Resonanzspektroskopie (NMR) voranzubringen, erlebte er einen Affront. Ramsey argumentierte kühl: „I think that some of your planned experiments are based on a misinterpretation of my result. The comment you added to your letter indicates that you were beginning to get a glimmer of the difficulty. “1 Gutowsky konnte nur ironisch parieren: „I am not sure that my glimmer of understanding has increased to a full 60 watt knowledge".2 
Ramseys Überlegenheitsgefühl schränkte die Möglichkeiten zur Kooperation des theoretischen Physikers und des experimentell arbeitenden Chemikers empfindlich ein. Gleichzeitig verstärkte es jedoch Gutowskys Bemühungen um Autonomie, indem er eine eigenständige Basis zur Interpretation der Messergebnisse ausfindig machte. Entsprechend trennten sich die Theorien der NMR. Zwar gelang es Ramsey, eine physikalische Erklärung des beobachteten Phänomens zu finden, diese ließ sich aber nur auf das einfachste Beispiel anwenden. Komplexere Fälle konnten nicht berechnet werden, sondern basierten auf empirischen Messungen und Abschätzungen, die nur mit Hilfe chemischer Theoriebildung verallgemeinert werden konnten. Dadurch kam es zur Ausbildung eines neuen, interdisziplinären Feldes, der chemischen NMR.

Wissenschaftler wie Gutowsky, die in der theoretischen Physik einen fachfremden Beitrag leisten wollten, erfuhren des öfteren Ausgrenzung, Ablehnung und Unverständnis. Wie im Folgenden gezeigt werden wird, spielten Emotionen und Affekte eine nicht zu unterschätzende Rolle in der Auseinandersetzung. Es werden dadurch „feine Unterschiede“ im Habitus der Vertreter der beteiligten Disziplinen erkennbar (Bourdieu 1999), Hierarchien der Disziplinen und Statusunterschiede innerhalb wissenschaftlicher Gemeinschaften werden so deutlich. Diese Abstufungen sollen hier weder normativ verstanden werden, noch ist ihre Genese Gegenstand meiner Untersuchung. Sie dienen vielmehr dem historischen Verstehen mancher der Abläufe und Widerstände, die bei der Ausbildung neuer wissenschaftlicher Felder auftraten.

Der Transformation von Methoden kommt dabei eine herausragende Bedeutung zu. Forschungsmethoden bestimmen mit, welche Phänomene als wissenschaftliche Objekte gelten können. Dadurch tragen sie auch dazu bei, neue Forschungsfelder zu schaffen. Es geschieht allerdings selten, dass eine Disziplin oder Forschungsrichtung auf einer einzigen Methode begründet wird. Oft stammen die Methoden aus verschiedenen Bereichen und müssen neu kombiniert werden. Die Materialwissenschaften können als ein Paradebeispiel dafür gelten, wie Methoden verschiedener Disziplinen für die Bildung eines neuen Forschungsfeldes eingesetzt werden. Dabei ist es wichtig zu untersuchen, ob eine Integration verschiedener Methoden stattgefunden hat, inwieweit es zum Wissenstransfer zwischen den beteiligten Disziplinen gekommen ist, und ob eine neue, selbstständige und als solche identifizierbare Forschungsrichtung entstanden ist (Bensaude-Vincent 2001, Hentschel 2010).

Welche Prozesse laufen ab, wenn ursprünglich für bestimmte Objektbereiche geschaffene Forschungsmethoden in anderen Zusammenhängen eingesetzt werden? Wie kommt es zur Stabilisierung der neuen Forschungsfelder? Vieles an diesen Transfer- und Transformationsvorgängen wurde bereits untersucht, allerdings eher in wissenssoziologischer und epistemologischer Hinsicht (Lynch/Jasanoff 1998, Jordan/Lynch 1998, Reinhardt 
2006a). Nur wenig ist darüber bekannt, wie die Vertreter bestimmter Disziplinen in ihrem Verhalten auf Veränderungen in ihrer Methodik reagieren und welche Gruppenbildungsprozesse dabei ablaufen. Mary Jo Nye hat in ihren Analysen zur Geschichte der theoretischen Chemie gezeigt, dass Identifikationsvorgänge mit bestimmten Instrumenten, Laboratorien und sozialen Strukturen für die Ausbildung einer disziplinären Identität unabdingbar sind (Nye 1993: 27-29, 266-269). Am Beispiel der neuesten Geschichte der theoretischen Chemie hat wiederum Buhm Soon Park deutlich gemacht, dass die Identifizierung der Akteure mit der Forschungstechnologie des Computers entscheidend zur Disziplinenbildung und auch ihrer Aufspaltung beitragen kann (Park 2003). Nye hat die Analogie der disziplinären mit der nationalen Identität betont und dadurch Aspekte in den Vordergrund gestellt, die bis in den Bereich des kollektiven Gedächtnisses reichen, der Basis der kollektiven Identität (Straub 1998). Der Schwerpunkt der neueren soziologischen Identitätsforschung liegt auf gesteuerten Erinnerungs- und Vergessensprozessen, die zur Aus- und Abgrenzung sozialer Gruppen führen. Diesem folgend untersuchen Historiker hauptsächlich politisch eingesetzte Strategien der kollektiven Identitätsbildung (Niethammer 2000). Gleichzeitig wurde dabei auch erkannt, dass die Anwendung einer psychologischen Theorie, die der Erklärung der personalen Identität und ihrer Krisen im Lebenslauf dient, auf Identitäten sozialer Gruppen problematisch ist (Straub 1998). Zwar lassen sich die Ansätze kollektiver Identitätsforschung auch für die Analyse der von einzelnen Akteuren bewusst gesteuerten politischen und sozialen Identifikationsprozesse einsetzen - doch für die Untersuchung impliziter Identifikationsschemata der Akteure mit bestimmten Methoden eignen sie sich nicht. Dabei betont Nye selbst die Ähnlichkeit ihres Verständnisses der disziplinären Identität mit dem Begriff des Denkstils von Ludwik Fleck (Nye 1993: 20-21) und bringt dadurch Aspekte eines impliziten, praktischen Wissens ins Spiel, das die Wahrnehmungs- und Denkschemata einer Gruppe prägt. Flecks Begrifflichkeit unterstreicht die von den Akteuren nicht bewusst hinterfragten Aspekte kollektiven Denkens, wobei ein Denkstil auch aus- und abschließend wirken kann. Für Fleck spielen Denkkollektive, die einen bestimmten Denkstil aufweisen und diesen auch erzwingen, die entscheidende Rolle in der Wissenschaft (Fleck 1980 [1935]). Nicht im Detail untersucht aber hat er die Prozesse des Aufeinandertreffens zweier Denkstile, die, obwohl sie unterschiedliche methodische Zugänge vertreten, dennoch fusionieren. In Anlehnung an Fleck hat Peter Heering einen spezifischen Experimentierstil postuliert, der die Bedeutung experimentellen Arbeitens in den Vordergrund rückt (Heering 2007).

Fokussieren wir auf Forschungsmethoden und ihre Einbettung in neue Forschungszusammenhänge - beziehungsweise auf das Entstehen neuer Forschungsbereiche durch Transformation bestehender Forschungstechnologien - so sehen sich die Wissenschaftler vereinfacht gesehen oft mit zwei 
Denkstilen konfrontiert, und zwar dem des disziplinären Zusammenhanges, aus dem die jeweilige Forschungstechnologie stammt und dem des Bereiches, in den diese Technologie mittels neuer Methoden eingeführt werden soll. Viele der Forschungsinstrumente des 20. Jahrhunderts besitzen ihre Basis in der Physik, deren Status als Leitwissenschaft nach 1945 vielleicht in ethischer, aber nicht in epistemologischer Hinsicht gefährdet war. Die Physik versprach, und verspricht immer noch, grundlegende, exakte und nachprüfbare Erklärungen für Problemstellungen auch anderer Disziplinen zu liefern. Sie gilt als "harte" Wissenschaft und nahm zumindest noch in den 1960er und 1970er Jahren die Spitzenposition in der "Pyramide“ der Wissenschaften ein (Smith u. a. 2000). Die Untersuchungsgegenstände dieses Artikels, NMR und Massenspektrometrie, sind auf breiter Front in den Natur- und Technikwissenschaften eingesetzte physikalische Forschungsmethoden. Charakteristisch ist ihre Zwischenstellung zwischen der Physik und der Chemie. Gilt die Physik von der Tendenz als grundlagenorientiert, mathematisch und auf allgemeine Gesetzmäßigkeiten ausgerichtet, so wird die Chemie eher als anwendungsorientiert, weniger mathematisch und auf Einzelfälle ausgerichtet beschrieben (Nye 1993: 278-283). Beide Disziplinen und die genannten Forschungstechnologien gehören zu den Bereichen, aus denen sich die Materialwissenschaften zusammensetzen. Die Probleme, die sich an den Beispielen der Methodenentwicklung von NMR und Massenspektrometrie aufzeigen lassen, sollten demnach auch bei der Ausbildung der Materialwissenschaften zu beobachten sein. Mit ihrer Einführung ist ein de-skilling der bis dahin die Methoden beherrschenden Elite in einer Disziplin verbunden. Diese Prozesse werden oft als Bedrohung wahrgenommen und sie gefährden bestehende Hierarchien, sowohl innerhalb von Forschergruppen als auch zwischen den Disziplinen (Johnson 1989).

Grundlage meiner Untersuchung sind Selbstbeschreibungen und Stellungnahmen von Wissenschaftlern, wobei diese meist retrospektiv gemacht wurden und oft emotionale Äußerungen über Exklusion, Andersartigkeit und Konflikte beinhalten. Um einen Zugang zu diesen Quellen zu finden, stütze ich mich auf ein Konzept, das Unterschiede zwischen Gruppen mit internalisierten Verhaltensweisen und verschiedenen Wahrnehmungen der sozialen Wirklichkeit korreliert: die Begriffe des Feldes und des Habitus von Pierre Bourdieu. Für Bourdieu ist ein Feld ein Möglichkeitsraum für Handlungen jeweils spezifischer Akteure, seien es Literaten, Künstler, Juristen oder Wissenschaftler. Jedes Feld hat besondere soziale Strukturen, die durch die Verteilung symbolischen Kapitals bestimmt werden.

Bourdieu hat seine Theorie nicht eigens für die Analyse der Wissenschaft entwickelt. Er analysiert vielmehr Klassenunterscheidungen anhand der „feinen Unterschiede" des Lebensstils und dessen ökonomischer, sozialer und kultureller Grundlagen (Bourdieu 1999 [1979]). Sein wichtigstes Beispiel ist die Nachahmung des Habitus des Bildungs- und Großbürgertums durch das 
aufstrebende Kleinbürgertum. Zugehörige $\mathrm{zu}$ einer bestimmten Klasse zeichnen sich stärker durch bestimmte Habitusformen als durch ihre tatsächliche soziale Positionierung aus. Diese Formen sieht Bourdieu

als Systeme dauerhafter und übertragbarer Dispositionen, als strukturierte Strukturen, die wie geschaffen sind, als strukturierende Strukturen zu fungieren, d.h. als Erzeugungs- und Ordnungsgrundlagen für Praktiken und Vorstellungen, die objektiv an ihr Ziel angepasst sein können, ohne jedoch bewußtes Anstreben von Zwecken und ausdrückliche Beherrschung der zu deren Erreichung erforderlichen Operationen vorauszusetzen, [...] und genau deswegen kollektiv aufeinander abgestimmt sind, ohne aus dem ordnenden Handeln eines Dirigenten hervorgegangen zu sein. (Bourdieu 1993 [1986]: 98-99)

Die von Bourdieu als Habitus bezeichneten erworbenen Dispositionen stellen die Internalisierung der Regeln des Feldes dar. Der Habitus wird durch Erziehung, Ausbildung und Sozialisierung erworben und kann im Einklang mit oder im Widerstand zu den Kräften des Feldes stehen. Er trägt so entweder zur Bewahrung oder zur Veränderung der Feldstrukturen, also der Machtverteilung, bei (Bourdieu 1998 [1980]: 24-25). Hauptsächlich dient er aber der Stabilisierung bestehender Strukturen: „Da er ein erworbenes System von Erzeugungsschemata ist, können mit dem Habitus alle Gedanken, Wahrnehmungen und Handlungen, und nur diese, frei hervorgebracht werden, die innerhalb der Grenzen der besonderen Bedingungen seiner eigenen Hervorbringung liegen.“ (Ebd.: 102) Als „subjektives, aber nichtindividuelles System verinnerlichter Strukturen, gemeinsamer Wahrnehmungs-, Denk- und Handlungsschemata“ sorgt der Habitus für die „objektive Abstimmung der Praktiken und die Einheitlichkeit der Weltanschauung mit der vollkommenen Personenungebundenheit und Substituierbarkeit der Praktiken und Einzelanschauungen" (ebd.: 112). Bourdieus Konzept betont sowohl die Einschreibung des Habitus in den Körper als auch dessen kognitive Dimension. Eine von Bourdieu doxa genannte, nicht hinterfragte und als selbstverständlich angenommene Weltsicht ergänzt das Habitus-Konzept mit einem, so der Wissenssoziologe Hubert Knoblauch, ,ausgeprägt emotionalen Charakter. Essentielle Sicherheit und Angst sind unmittelbar mit der Ordnung des Selbstverständlichen verknüpft" (Knoblauch 2005: 221). Darüber hinaus ist der Habitus durch Körperwahrnehmungen geprägt, „feste Assoziationen von schon bekannten Situationen und Empfindungen, die kulturell oder auch individuell eine Bedeutung tragen." In ihrer kognitiven und sozialen Bedeutung können diese assoziativ gesteuerten Erinnerungen jederzeit aufgerufen werden, wobei diese Prozesse sowohl soziologisch als auch neurophysiologisch dargestellt worden sind (Diehl 2005: 173-175).

Nimmt man die kognitiven, sozialen und körperlichen Bedeutungsebenen des Habitus zusammen und bezieht sie auf die Wissenschaften, so erkennt man, dass der Bourdieu'sche Habitus-Begriff dem Fleck'schen Denkstil übergeordnet ist. Letzterer stellt die kognitiv-soziale Komponente des Habitus dar. 
Das spezifische Kriterium des wissenschaftlichen Feldes, zum Beispiel im Vergleich zum ökonomischen, ist nach Bourdieu die als Eintrittsvoraussetzung geforderte Uneigennützigkeit seiner Vertreter. Trotzdem treten heftige innere Verteilungskämpfe um wissenschaftliches Kapital auf, für deren Bewertung Wissenschaftler „auf das Urteil“ verweisen, „das die Erfahrung verhängt, auf das ,Wirkliche، “ Dabei zeichnen sich Wissenschaftler durch den Grad der Einigkeit aus, mit dem sie sich über Grundsätze der Bewertung und der anzuwendenden Methoden verständigen, „über den stillschweigenden, untrennbar politischen und kognitiven Vertrag, der die ,Arbeit der Objektivierung" begründet und beherrscht“. Dies verhindert nicht, dass sich verschiedene Repräsentationen einander gegenüberstehen können, wobei sie „mit dem gesamten, kollektiv angehäuften und kollektiv angewandten Arsenal von Methoden, Instrumenten, Techniken, unter dem Druck der Disziplinierungen und Zensuren des Feldes und unter Mithilfe jener unsichtbaren Tugend einer Orchestrierung der habitus" vertreten werden (Bourdieu 1998: 29 f.).

Die durch die Akkumulation wissenschaftlichen Kapitals gebildete Autorität einzelner Wissenschaftler oder Gruppen ermächtigt diese dazu, in das wissenschaftliche ,Spiel' einzugreifen und gegebenenfalls sogar die Spielregeln zu ändern. Zu den Eingriffen gehören Wechsel der Forschungsthemen, der Methoden oder der Publikationsorte. Wissenschaftler, die in das jeweilige Feld ,hineingeboren' wurden, verfügen über einen ausgeprägten ,Spielsinn', eine erworbene Eigenschaft, die es ihnen erlaubt, besonders erfolgreich am Spiel teilzunehmen. Wissenschaftliches Kapital kann nach Bourdieu in zwei Arten eingeteilt werden: eine, die im Wesentlichen auf der administrativen Position des jeweiligen Akteurs beruht und eine andere, die sich auf die wissenschaftliche Reputation im engeren Sinn stützt. Beide Kapitalarten können an ein und derselben Institution vertreten sein, sie repräsentieren aber unterschiedliche Karrierestrategien.

Während Bourdieu im Wesentlichen zwei Gruppen untersucht, die unterschiedliche Strategien zum Ansammeln von Kapital verfolgen, die an Macht orientierten Wissenschaftsmanager und die auf Reputation ausgerichteten Wissenschaftler (Bourdieu 1998), und Terry Shinn auf die Unterschiede in den Forschungsstrategien verweist, die mit professionellen Rangunterschieden der Forscher zusammenhängen (Shinn 1988), so möchte ich hier Gruppierungen betrachten, die sich durch die Art und Weise der Gewinnung, Begründung und Darstellung wissenschaftlichen Wissens unterscheiden. Mit den Physikern, die die Theorie der Methode und die Instrumente liefern, sowie den Chemikern, die die Anwendungsprobleme stellen, treffen Vertreter zweier unterschiedlicher Habitusformen aufeinander. Sicher weichen diese nicht grundsätzlich voneinander ab. Aber innerhalb des wissenschaftlichen Feldes verweisen sie doch auf „feine Unterschiede“ zwischen Physik und Chemie. Treffen unterschiedliche Richtungen aufeinander, 
so sollte dies, folgt man Bourdieu, an der „Orchestrierung“ der Habitusformen zu erkennen sein, deren Aufeinandertreffen die Forscher durch die gegenseitige Infragestellung der stillschweigend anerkannten Arbeit der Objektivierung verunsichert und damit auch emotional geäußerte Reaktionen nach sich zieht.

Für die Zielrichtung dieses Artikels liegen die Vorteile der Bourdieu'schen Theorie in der Korrelation von Gruppenzugehörigkeit und Darstellungsformen der wissenschaftlichen Objekte sowie in dem von Bourdieu konstatierten Bemühen sozialer Gruppen, den Habitus einer anderen Gruppe zu imitieren. Ein weiterer Vorteil ist die Möglichkeit, Äußerungen emotionalen und affektiven Charakters für die Interpretation heranzuziehen. Ein Nachteil ist die mangelnde Erklärbarkeit von Veränderungen: Bourdieus Konzept betont das Festhalten am Status quo, obwohl es durch die Versuche der unteren Klassen, die oberen $\mathrm{zu}$ erreichen, beziehungsweise $\mathrm{zu}$ imitieren, eine dynamische Komponente enthält. Die Übernahme physikalischer Methoden in der Chemie sehe ich hier in Analogie dazu, wobei ich davon ausgehe, dass die Physik über weite Bereiche des 19. und 20. Jahrhunderts tonangebend war. Theoriefähigkeit und Mathematisierbarkeit, Beherrschung und Weiterentwicklung des Instruments, sowie das Prestige in einer breiten Öffentlichkeit stehen für die Physik. Auf der anderen Seite steht die Bereitstellung der Forschungsfragen und der Forschungsmittel für die Chemie, vor allem für die organische Chemie als dominierende Subdisziplin. Die organische Chemie fungierte als Gegengewicht zur Physik und wies starke Beharrungstendenzen auf. Dabei sind auch die Grenzen der Analogie von Disziplinen und Klassen zu sehen.

Der Beitrag untersucht intra- und interdisziplinäre Dynamiken, die durch den Transfer von Methoden ausgelöst werden. Diese Dynamiken reichen von Negierung neuer Methoden über innerdisziplinäre Transformation bis zur Ausdifferenzierung neuer Felder. Mit der hier beispielhaft untersuchten Einführung physikalischer Methoden in die Chemie entstand ein neues wissenschaftliches Feld, dessen Schwerpunkt in der Entwicklung von Methoden lag (Reinhardt 2006a). Die „Methoden-Macher“ entwickelten, so die These, einen eigenen, spezifischen Habitus, sie gingen zwischen „Organikern“ und „Theoretikern“ neue Wege. Diese Ausdifferenzierung stellte die Unterschiede von Physik und Chemie nicht grundsätzlich in Frage. Sie fügte ein neues, eigenständiges Feld ein, das Brücken zu weiteren Bereichen, wie den Biowissenschaften, der Medizin und den Materialwissenschaften ausbildete und sich so weiter stabilisierte. Die Frage, ob die Bildung neuer wissenschaftlicher Felder, die je durch spezifische Habitusformen geprägt sind, allgemein aus Grenzkonflikten erklärbar wäre, lässt sich in diesem Beitrag nicht abschließend beantworten. Es werden aber Dynamiken deutlich, die auch in anderen Fällen erkennbar sein sollten.

Die Beobachtung hierarchischer Beziehungen zwischen Physikern und Chemikern ist keineswegs selbstverständlich und sicher auch zeitgebunden. 
Dennoch weisen, neben den Klassifikationsversuchen Comte'scher Prägung und den Image-Zuschreibungen in der Öffentlichkeit, Äußerungen und Handlungen von Chemikern und Physikern darauf hin, dass es in der Mitte des 20. Jahrhunderts die weiter unten beschriebenen Hierarchieunterschiede gegeben hat. Diese „feinen Unterschiede“ sollten die Art der Übertragung von Methoden sowie der Ausbildung eines neuen Forschungsbereiches bestimmen, eine Transformation, die hier unter dem Begriff der Physikalisierung kurz beschrieben werden soll. ${ }^{3}$

\section{Physikalisierungen}

Chemie und Physik haben - bei allen Ähnlichkeiten, die natürlich auch zu beobachten sind - Unterschiede aufzuweisen. Nicht nur feinsinnige Beobachter des Wissenschaftsbetriebs wie der Karikaturist Sidney Harris haben dies festgestellt (vgl. Abb. 1). Auch die Akteure selbst haben die Überwindung dieser Hindernisse thematisiert. In Abbildung 2 ist ein Chemiker zu sehen, der mit einem Reagenzglas in der Hand über einen Magneten springt. Das Reagenzglas dient als Symbol für die klassische chemische Methode, die chemische Reaktion. Der Magnet dagegen steht für moderne Methoden in Gestalt der physikalischen Techniken. Der Wissenschaftler ist die Allegorie eines Forschers, der die Hürde von den klassischen chemischen zu den modernen physikalischen Methoden nimmt, dabei als Vorbild dient und somit den Weg für andere nach ihm ebnet. Dieses Bild, das einem technischen Bulletin des wichtigsten Herstellers von NMR-Geräten in den 1950er und 1960er Jahren, Varian Associates, entnommen ist, zeigt, dass die Beteiligten, die zwischen den etablierten Bereichen standen, diese Kluft deutlich wahrnahmen und dass sie damit warben, sie überwinden zu können.

Versuche, diese Kluft zu überbrücken, begleiteten einen Prozess, den ich hier als Physikalisierung bezeichnen möchte und der auf verschiedenen Ebenen wissenschaftlicher Tätigkeit ablief. In meiner Definition bedeutet Physikalisierung die Übernahme von ursprünglich in der Physik entwickelten Forschungsmethoden durch andere wissenschaftliche Disziplinen. Zweck der Methoden ist die Erzeugung numerischer Daten, ihr Kernbereich besteht in der Manipulation physikalischer Parameter und Objekte, wie dem Elektron und dem Atom. Objekte dieser Art werden mit Hilfe experimenteller Techniken genauer bestimmt beziehungsweise vermessen und mittels mathematisierter Theorien beschrieben. Sie besitzen in den aufnehmenden Disziplinen allerdings oft einen anderen Stellenwert als in der Physik selbst. Mit der Physikalisierung verbunden, aber davon abzugrenzen, ist eine Mathematisierung und Technisierung der Wissenschaften. In die Physikalisierung eingeschlossen sind Modellbildungen, Veranschaulichungen oder Visualisierungen wissenschaftlicher Objekte. Weiter werden bestimmte Repräsentationsformen und 


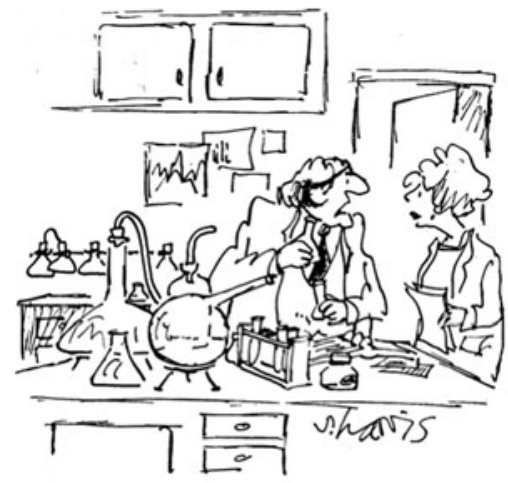

"I'M ON THE VERGE OF A MAJOR BREAKTHROUG BUT IM ALSO AT THAT PONTT WHERE CHEMISTRY LEANES OFF AND PHYSKS BEGINS, SO I'LL HAVE TO DROP THT WHTLE THING:

Abb. 1 Cartoon von Sidney Harris (Nye 1993:

Frontispiz). Copyright ScienceCartoonsPlus.com

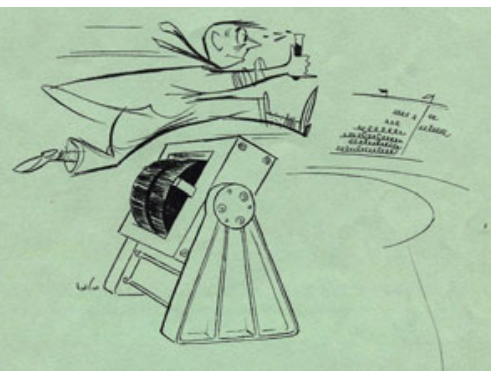

Abb. 2 Teil der Titelseite einer Einlage des Technical Information Bulletin, 2 (1958), 2, von Varian Associates. Illustration use courtesy of Agilent Technologies

Metaphern übernommen. Die Physikalisierung hat demzufolge tiefgreifende Auswirkungen auf den Stil, die Organisationsform und den Habitus der Wissenschaften. Historisch gesehen, beschreibt die Physikalisierung Wandlungsprozesse, die seit dem Ende des 18. Jahrhunderts zu beobachten sind, ohne aber jemals vollständig andere Disziplinen grundsätzlich umgestellt zu haben. Als frühes Beispiel kann hier die Lavoisier'sche Revolution der Chemie in den 1780er und 1790er Jahren gelten (Perrin 1988). Oft kommt es bei der Übernahme physikalischer Methoden aber auch zu einer Transformation, die in die Stärkung des schon vorher bestehenden Denkstils mündet. Es handelt sich also um einen wechselseitigen Prozess, der auch eine Veränderung der Physik selbst herbeiführen kann. Darüber hinaus sind diverse Überträgerstufen festzustellen, die in manchen Fällen einen direkten Kontakt zwischen Vertretern der Physik und der aufnehmenden Disziplinen unnötig machen. Dies können zum Beispiel Instrumentenhersteller oder Hybriddisziplinen sein (Reinhardt 2004).

Dennoch kommt es auf verschiedenen Ebenen zu einer Umformung der aufnehmenden Disziplin (Reinhardt 2007). Im Fall der Physikalisierung der Chemie durch spektroskopische Methoden in der Zeit von etwa 1930 bis 1960 veränderten die Chemiker zunächst die Objektebene. Dabei kam es zu einem Übergang von einer auf Stoffen und ihren Reaktionen basierenden Chemie zu einer Chemie, die molekulare Strukturen und ihre Dynamiken in den Mittelpunkt stellte. Begonnen hatte dieser Wandel bereits Ende des 19. Jahrhunderts. Im Laufe des 20. Jahrhunderts wurden Stoffe durch abstrakte Modelle ersetzt (Schummer 2002, Slater 2002). Auf der zweiten, der methodischen Ebene erfolgte eine Verdrängung chemischer Reaktionen durch 
physikalische beziehungsweise technische Methoden. Diese Methoden sind, ganz im Sinne der "genericity“ der „research technology“ Shinns (Joerges/ Shinn 2001), auf ähnliche Weise in vielfältigen Anwendungsfeldern einsetzbar. Diese zunehmende Abstraktion und ihre Erfassung mit neuen Methoden war Bedingung der Expansion des mentalen Modells der Chemie, des Denkens in Strukturen (Steinhauser 2009), in weitere Bereiche, darunter auch die Materialwissenschaften. Als Folge dieser beiden Transformationsebenen ist $\mathrm{zu}$ konstatieren, dass die epistemologischen Unterschiede zwischen den Disziplinen zu verschwimmen begannen. Auch auf der dritten Ebene, der der sozialen Organisation und handwerklichen Laborpraxis, kam es zu beträchtlichen Verschiebungen: Qualifizierte Chemiker, die bis dahin für die aufwändige Forschungsarbeit zuständig waren, wurden in bestimmten Aufgabenbereichen durch Instrumente ersetzt. Da die vertraute Laborumgebung einschließlich der Kompetenzzuschreibungen im Umbruch war, gefährdete dies die Identität der Chemiker durch de-skilling und die Konkurrenz mit Vertretern anderer Disziplinen. Eine direkte Gegenüberstellung eines typischen Labors der 1930er Jahre und eines weit verbreiteten Instruments der 1960er Jahre soll das Ausmaß der Transformation veranschaulichen. Bestand in dem hier gezeigten Labor des Münchner Naturstoffchemikers Hans Fischer die Laborarbeit der Chemiker zu einem großen Teil in der Durchführung chemischer Reaktionen mit Stoffen, so war dies in der NMR-Spektroskopie der 1960er Jahre weder nötig noch möglich. (vgl. Abb. 3 und 4) Dies hatte auch beträchtliche Verschiebungen der Hierarchie innerhalb der Chemie sowie zwischen Chemie und weiteren beteiligten Disziplinen zur Folge: Lag bis dahin der Hauptteil der Arbeit bei einer großen Zahl abhängig beschäftigter Doktoranden und Postdoktoranden und damit die Kompetenz des Laborleiters auf einer prinzipiell gleichen Ebene, so waren nun hochqualifizierte Fachwissenschaftler benachbarter Bereiche gefragt (wie etwa der Physik oder der physikalischen Chemie), die nicht subordiniert waren und zudem über ganz andere Kompetenzen als die Nutzer verfügten.

\section{Reaktionen und Strategien}

Der Begriff ,physikalische Methoden“ wurde zu Anfang des 20. Jahrhunderts geprägt. 1912 unterschied der physiologische Chemiker Emil Abderhalden in seinem Praktikumshandbuch chemische und physikalische Methoden. Etwa gleichzeitig erschienen Titel in der analytischen und der physikalischen Chemie, die den Begriff weiter verbreiteten. In der Organischen Chemie ist Arnold Weissbergers „Physical Methods of Organic Chemistry“ zu nennen, das 1945 erschien. Das wichtigste Handbuch über Methoden der Organischen Chemie von Heinrich J. Houben und Theodor Weyl enthält 1955 auch Bände über physikalische Methoden. ${ }^{4}$ Die Begrifflichkeit tauchte zunächst an den 


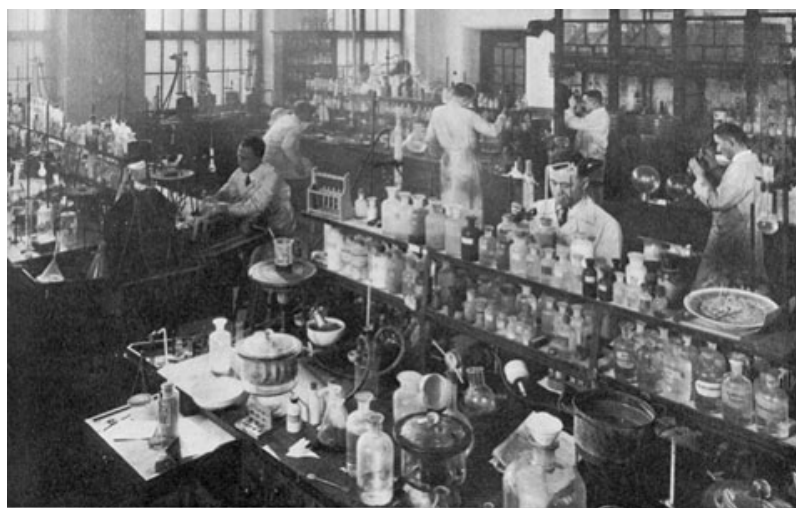

Abb. 3 Mitarbeiter Hans Fischers bei der Laborarbeit, ca. 1935 (Treibs 1971: 61)

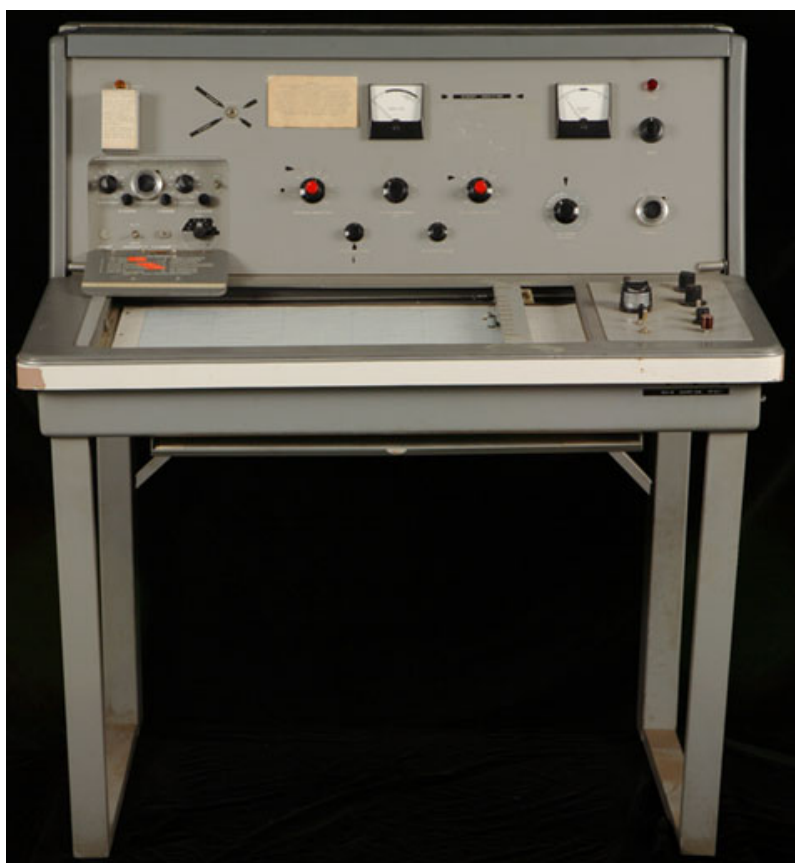

Abb. 4 Konsole des 1961 auf den Markt gebrachten Varian A-60 NMR-Spektrometers, das für Routinemessungen hauptsächlich in der Organischen Chemie genutzt wurde (Courtesy of Chemical Heritage Foundation Collections, Philadelphia, USA. Fotografie von Gregory Tobias)

Rändern auf, in angewandten Bereichen, wie der Analyse von Lebensmitteln, der Physiologie oder der Medizin, und brachte oft eine Diskrepanz zwischen chemischen und physikalischen Methoden mit sich. Schon die Bezeichnung ,physikalische Methoden ' trug ein ganzes Bündel von Bedeutungen: Physik galt als die fundamentale Disziplin, als modern und Hightech. Aber der Begriff 
drückt auch die Fremdartigkeit der neuen Methoden aus, eine Fremdartigkeit, die einen Verlust an Vertrautheit beschrieb - mit der gewohnten Laborumgebung, den eingeübten Handgriffen und den wahrgenommenen Gerüchen.

Im Folgenden wird eine Reihe von Reaktionen der Physiker, Chemiker und beteiligten Methodenentwickler beschrieben, die von Opposition bis zu kompletter Aneignung reichen. Diese Reaktionen führten einerseits zu Versuchen, jegliche physikalische Bedeutung der Methoden zu entfernen, riefen aber andererseits auch Taktiken hervor, mit denen physikalische Konzepte und Theorien quasi huckepack mit den Instrumenten und Methoden in die Chemie transferiert wurden. Mein Argument ist schließlich, dass ein Teil der Chemiker einen Vorteil darin sah, Methoden für ihre Kollegen zu entwickeln und weiterzuverbreiten. Diese Methodenmacher bildeten eine eigene Gemeinschaft, die Brücken nicht nur zwischen Physik und Chemie, sondern auch zur Medizin, den Lebenswissenschaften und den Materialwissenschaften schlug (Reinhardt 2006a).

Die folgenden Schilderungen und Zwischenfälle vermitteln etwas von den Vorurteilen der Akteure. Die eingenommene Perspektive ist die der Wissenschaftler, die die neuen Methoden entwickelten und in der Chemie verbreiteten. Ihre Handlungen und Erfahrungen dienen hier als Spiegel für die Reaktionen der ,abgebenden' und der ,aufnehmenden' Wissenschaftlergemeinschaften. ${ }^{5}$

\section{Unterlegenheit und Eigenständigkeit}

In der zweiten Hälfte des 20. Jahrhunderts bildete eine ausgefeilte Elektronik die technische Basis physikalischer Methoden, und die Instrumente wurden meistens von einer spezialisierten Industrie hergestellt. Man könnte also annehmen, es bestünde keine Notwendigkeit einer direkten Kontaktzone zwischen Physik und Chemie. Allerdings gab es diese durchaus, zum Beispiel in Form der Zwischendisziplin Chemical Physics, die in den späten 1920er Jahren entstand und die die neu von der Quantenphysik aufgebrachten Probleme durch molekülspektroskopische Experimente lösen wollte. Hier arbeiteten Chemiker und Physiker direkt zusammen (Reinhardt 2004). 1947 wurde Herbert Gutowsky, ein junger physikalischer Chemiker an der Harvard University in die NMR eingeführt. Sein Tutor war der Physiker George Pake, ein Mitarbeiter in der Arbeitsgruppe Edward Purcells, dem nur etwa ein Jahr zuvor die Beobachtung des der NMR zugrundeliegenden physikalischen Effekts gelungen war.

1947 war NMR noch eine Experimentaltechnik, die vor allem dazu diente, genaue Messungen von magnetischen Kernmomenten durchzuführen, und sie stellte eine der wichtigsten neuen Methoden der Kernphysik dar. Pake und Gutowsky interessierten sich allerdings mehr für Strukturen von Festkörpern, in Einklang mit einem der Themengebiete der Gruppe um Purcell. Auch innerhalb dieses Arbeitsgebietes gab es charakteristische Unterschiede bei den theoretischen und experimentellen Zugängen: Pake zielte eher auf 
grundlegende Modelllösungen ab, während Gutowsky an Einzelfällen interessiert war. War Pake mit der Konstruktion eines theoretischen Modells zufrieden, begann für Gutowsky mit dessen Anwendung auf spezifische Fragen erst die eigentliche Arbeit (ebd.). Dabei spielte das theoretische Modell der Kernspinresonanz in Festkörpern eine Vermittlerrolle zu Fragen der Strukturforschung (vgl. Morgan/Morrison 1999). In der täglichen Zusammenarbeit von Pake und Gutowsky, die etwa gleichen Alters waren, zeichneten sich Statusunterschiede ab: Der Physiker Pake konstruierte das Spektrometer und nur er durfte es bedienen. Der Chemiker Gutowsky synthetisierte die Probesubstanzen. Die Kontrolle des Instruments war das entscheidende Signum für die Kontrolle über die Zusammenarbeit und das gesamte Forschungsfeld. Erst als Gutowsky an das Department of Chemistry der University of Illinois wechselte, konnte er mit der Kontrolle über ein selbstgebautes Spektrometer auch seine Selbstständigkeit sichern. In Illinois gelang ihm in der Folge die Aufklärung einiger Effekte, die die Basis für die chemische Anwendung von NMR darstellten. Doch trotz der auf experimenteller Ebene gewonnenen Eigenständigkeit fühlte sich Gutowsky gegenüber den theoretischen Physikern im Hintertreffen. So schilderte er in einem Interview seine Erinnerung an eine Begegnung mit dem theoretischen Physiker John Van Vleck:

I always felt that chemists were inferior to physicists. It reminds me of my attempt earlier in the game to describe the phenomenon of spin-spin coupling to Van Vleck and I chickened out. I was at a symposium on molecular spectroscopy at Ohio State University. [...] And Van Vleck was there. And I knew him from my Harvard days but I never really met him. And I remember three or four times trying to walk towards him and then turning around and fleeing. Because I was groping for a model to explain the structure that we had observed. ${ }^{6}$

Dieses Gefühl der Unterlegenheit war nicht nur auf die Hierarchie bezogen, obwohl es hier große Unterschiede gab: Van Vleck war Leiter des Department of Physics an der Harvard University. Gutowsky war dagegen zur gleichen Zeit einfacher Dozent in Illinois und früher Doktorand an der Harvard University gewesen. Der zentrale Punkt war die Fähigkeit, ein erklärendes Modell aufzustellen, das die Theorie der NMR mit den beobachteten neuen Phänomenen verbinden sollte. ${ }^{7}$ Die Fähigkeit zur Aufstellung eines solchen Modells war das Ziel des arrivierten Chemikers, eine Fähigkeit, deren Nichtbeherrschung zu einem Unterlegenheitsgefühl führen konnte. Statusunterschiede werden in der Äußerung im Interview emotional belegt, wobei der Chemiker sich den Habitus des Physikers aneignen wollte, ihm dies allerdings nicht vollständig gelang. Solche Versuche wurden auf Seiten der theoretischen Physiker auch manchmal abwehrend beantwortet, wie die eingangs wiedergegebene Auseinandersetzung zwischen Gutowsky und Norman Ramsey zeigt. 


\section{Unglauben und Anspruch auf Geltung}

Auch von Seiten der aufnehmenden Disziplin, in diesem Fall der organischen Chemie, erlebten die Methodenmacher Abwehrreaktionen. Diese waren allerdings eher mit dem Unglauben verbunden, dass die neuen Methoden den alten gleichwertig, beziehungsweise ihnen sogar überlegen wären. Hier war auf der Seite der Organiker die Angst verbreitet, ihre Expertise würde einem de-skilling unterzogen und damit die eigene Handlungskompetenz nichts mehr wert. Damit eng verbunden war die Sorge, traditionelle Überprüfungskriterien der Chemiker würden außer Kraft gesetzt, ohne dass sie die neuen Vorgänge kontrollieren könnten. Im Fall der Einführung der Massenspektrometrie in die Chemie konnte anfänglich tatsächlich niemand nachvollziehen, was mit den Molekülen in den Geräten geschah. Die mit Hilfe der Massenspektrometrie neu entdeckten Verbindungen konnte man nicht sehen, riechen, schmecken beziehungsweise mit etablierten Methoden charakterisieren oder kalibrieren. Solche geäußerten Abwehrhaltungen in Bezug auf die Einführung neuer Methoden sind in den Gutachten zu finden, die über Artikelmanuskripte erstellt wurden. Einer der Herausgeber des Journal of the American Chemical Society, Robert L. Autrey, schrieb als Antwort auf die Manuskripteinreichung des auf Massenspektrometrie spezialisierten Chemikers Klaus Biemann:

You have detected 20 compounds, but we question that you have 'isolated' 16 of them. There is no demonstration, other than by gas chromatography, of their purity. None of the usual criteria of purity have been applied; none of the compounds have been characterized by any of the classical means: there are no melting points, no analyses, few ultraviolet and no infrared spectra. The chemist or chemical taxonomist discovering one of these compounds in another plant may be quite unable to ascertain that he has in hand a known compound, as few institutions have available a mass spectrometer of the caliber required to obtain these data. $^{8}$

Biemann antwortete, dies sei eine Frage der Definition von Isolierung einer Substanz und betonte im Weiteren die Verantwortung, die die Massenspektroskopiker für die Durchführung und Interpretation der Experimente hätten. ${ }^{9}$ Es sei eben kein technisiertes, automatisches Verfahren, das die Chemiker ersetzen sollte. Vielmehr seien es Experten, die die Chemiker bei ihren Arbeiten unterstützten, und zwar handele es sich um die akademischen Massenspektroskopiker. Biemann appellierte also an das Vertrauen der Kollegen, von denen er als gleichberechtigt wahrgenommen werden wollte. Diese wiederum aber wollten die Kontrollmöglichkeiten über die Experimente nicht abgeben. Biemann argumentierte also nicht mit einer Änderung des traditionellen Habitus, sondern betonte seine Anknüpfung an den etablierten Habitus analytischer Chemiker, die die Verantwortlichkeit für präzises und genaues Arbeiten der Person eines Wissenschaftlers, und nicht einem Instrument, zuschrieben. 
Auf dem Spiel stand aber nicht nur, wer die Experimente durchführen konnte, sondern vor allem, was als gültiger Abschluss eines Experiments gelten sollte. Für die traditionell arbeitenden Chemiker war dies die Isolierung der Substanz in reiner Form und in einer Menge, die man sinnlich wahrnehmen und mit der man weitere Versuche durchführen konnte. Für die Vertreter der neuen Methoden war dies nicht mehr nötig beziehungsweise sogar überflüssig: Ihrer Meinung nach war es nun möglich, geringste stoffliche Mengen zu charakterisieren. Damit veränderte sich das Verhältnis zu chemischen Stoffen generell, da sie mit den neuen Methoden eben nicht mehr in stofflicher Form produziert werden konnten, um als real zu gelten. Das Objekt der Forschung war nun nicht mehr die Substanz, sondern deren Spur.

\section{Bedrohung: Einhegung und Ausbruch}

Wie reagierte die zahlenmäßig stärkste und dominierende Gruppe in der Chemie, die organischen Chemiker, auf die Herausforderung durch eine neue Expertengruppe? Immerhin stand ihre Autonomie auf dem Spiel. Nun entschieden nicht mehr Reaktionen im Reagenzglas, die von abhängigen Doktoranden durchgeführt wurden, über ihre Forschung, sondern komplizierte Apparate, die von einer fremdartigen Gruppe aus Ingenieuren, Physikern und physikalischen Chemikern bereitgestellt und bedient werden mussten. Die Theorie der Instrumente war noch in der Sprache der Physiker gehalten und damit unverständlich und unzugänglich. Eine Lösung dieses Problems stellte das Servicelabor dar: Hier konnte die Physik auf Abstand gehalten werden. Chemiker gaben ihre Substanzproben ab und erhielten im Gegenzug Spektren, manchmal auch mit einer Nachhilfestunde in Interpretation verbunden (Laszlo 2002). Es handelt sich also um eine Einhegung der neuen Techniken, die durchgeführt wurde, um die etablierte Hierarchie innerhalb der aufnehmenden Disziplin nicht zu gefährden.

Im Zweiten Weltkrieg war die Infrarotspektroskopie eine der wichtigen neuen Techniken geworden, vor allem im Bereich der US-amerikanischen Programme zur Produktion künstlichen Kautschuks (Rabkin 1987). In zahlreichen Chemiefakultäten wurde in der unmittelbaren Nachkriegszeit die Infrarotspektroskopie in Form einer Serviceeinheit eingeführt, so auch an der University of Illinois am Standort Urbana-Champaign. Gutowsky war dort ursprünglich von den organischen Chemikern angestellt worden, um Dienstleistungen auf dem Gebiet der Infrarotspektroskopie zu erbringen. Mit den oben erwähnten Arbeiten zu NMR machte er sich von den Organikern unabhängig und etablierte eine eigene Arbeitsgruppe in der physikalischen Chemie. Trotzdem versuchten die Organiker unter der Leitung ihres einflussreichen Direktors Robert Adams, ihn wieder in eine dienstleistende Funktion zu drängen, diesmal mit NMR. Im Interview erinnert er sich an eine Situation in diesem Zusammenhang: 
The department had a coffee habit of meeting at a greasy spot across the street and flipping coins, see who'd pay for a round of coffee. It served a very useful communications purpose. People informally get problems solved they can't solve at any other place. I was there and Adams's aid was next to me. He had a kind of confidential manner when he wanted to speak out something that was emotion laden and bent over and he said: 'Hey, Herb, why don't you go ahead and run samples on your NMR machine.' And my response was unfortunately spontaneous and unreserved, and I said: 'Hell, no, over my dead body.' It showed the miserability of some people in the service work. ${ }^{10}$

Beide Seiten sahen sich voneinander bedroht. Während die Gruppe der organischen Chemiker ihre Autonomie in der Handhabung von Methoden und deren Interpretation gefährdet sah, so war die Gruppe der Methodenmacher der Überzeugung, ihre Unabhängigkeit in der Wahl der Forschungsthemen stünde auf dem Spiel. Die allgemein verbreitete Reaktion des Mainstreams der Chemie war die Abdrängung der neuen Methoden in untergeordnete Servicebereiche. Nicht allen Spektroskopikern gelang der Ausbruch so leicht wie Herbert Gutowsky. Hier spiegelt sich eine Hierarchie innerhalb einer Disziplin wider, die nicht mit den akademischen Karrierestufen korrelierte und deren Forschungsergebnisse verschiedenen Ansprüchen und Anwendungszielen folgten. Diese Hierarchie, die in Urbana-Champaign die Organiker an der Spitze sah, musste nicht immer so aufgebaut sein, war aber auch nicht untypisch.

\section{Beleidigung, oder wie man seine intellektuelle Ehre bewahrt}

Carl Djerassi verglich seine Wissenschaft, die Naturstoffchemie, einmal mit der Lage von jemandem, der in einem total abgedunkelten Zimmer Möbel erkennen sollte. Das Tasten, mit dem die Person herausfindet, ob es sich um Tische, Stühle oder andere Möbelstücke handelt, verglich er mit den von den Chemikern traditionell benutzten analytischen Reaktionen. Die neuen physikalischen Methoden brachten für Djerassi einen technischen Fortschritt: Sie seien wie Taschenlampen, die jeweils bestimmte Möbelstücke erkennbar machten. So bliebe ein Teil der intellektuellen Faszination erhalten. Die Forschungsfrage könne nur gelöst werden, indem man die einzelnen „beleuchteten Ausschnitte“ miteinander kombiniere, und diese gleichzeitig auch mit klassischen chemischen Reaktionen verbände. Ganz im Gegensatz dazu stand die von Djerassi als „flash-camera“ bezeichnete Röntgenkristallographie, die die dreidimensionale Struktur eines Moleküls auf einmal sichtbar machte. Sie verwandelte die Aufklärung der Strukturen von Naturstoffen in ein Routineverfahren:

In my opinion a number of people didn't use it [X-ray crystallography] because it was an insult. [...] If anyone can prove a structure with an X-ray analysis, we are nothing. The organic chemist is nothing but a little technician who crystallizes the compound and gives it to someone who sticks it in an X-ray machine, and even the rest is computerized. So what's your function? I would like to see if I can prove a 
structure without X-ray, but otherwise use everything else. [...] I think there was [a strong tendency] [...] to show "how clever I am using intellectual techniques. ${ }^{11}$

Djerassi lehnte die Methode der Röntgenkristallographie ab, weil er nicht in die Rolle eines einfachen Technikers gedrängt werden wollte. Aber er benutzte die anderen „flash-lights“ mit großer Begeisterung. Sie stellten eine intellektuelle Herausforderung per se dar, und die Versuche, sie für die chemische Praxis geeignet $\mathrm{zu}$ machen, machten sie noch attraktiver. Eine der neuen physikalischen Methoden, die Röntgenkristallographie, beschränkte die Handlungsfähigkeit der sie anwendenden Wissenschaftler aber auf eine Weise, dass sie sich in ihrer intellektuellen Ehre gekränkt sahen. Diese lag in der freien, schöpferischen Kombination der Informationen und ihrer kreativen Verschränkung. Die Röntgenkristallographie wiederum trieb das de-skilling so weit, dass die Strukturaufklärung eher einer technischen als einer wissenschaftlichen Aktivität glich. Hier war der Habitus der Wissenschaftler als Gesamtgruppe in Frage gestellt und ihr Selbstverständnis herausgefordert. Auch wenn im weiteren Verlauf der Geschichte die Verwendung der Röntgenkristallographie nicht mit dem Stigma der Unwissenschaftlichkeit belegt wurde, so zeigt doch diese Reaktion, dass auch intellektuelle Eitelkeit und Stolz eine Rolle bei der Akzeptanz bestimmter Methoden spielen konnten.

\section{Andersartigkeit: Mimikry und die Huckepack-Strategie}

Um physikalische Methoden für möglichst viele Chemiker anwendbar zu machen, benutzten ihre Proponenten die Strategie zu behaupten, bei diesen Methoden handele es sich nicht um Physik, sondern um Chemie. So vertrat Klaus Biemann diese Meinung, als er prognostizierte, die Vernachlässigung der Massenspektrometrie durch die Chemiker würde sich ändern, wenn die Chemiker erkennen würden, dass sie nicht nur ein kompliziertes Instrument sei, sondern auch eine Abbaureaktion, mit der automatisch die Produkte dieser Reaktion getrennt und deren Molekularmasse bestimmt werden könnte (Reinhardt 2006a: 112 f.). In dieser Argumentation wird die Herstellung von Bezügen der neuen, physikalischen Methoden zu den klassischen, chemischen Reaktionen besonders gut sichtbar. Die neuen wurden als Chemie repräsentiert.

Es gab auch andere Ansätze. Einige davon waren fundamental-physikalisch und mathematisch und daher für einen großen Teil der Chemiker nur schwer anwendbar. Andere waren so pragmatisch, dass sie nicht einmal das Verständnis der einfachsten physikalischen Grundlagen der eingesetzten Methoden voraussetzten. In der Mitte zwischen diesen beiden Extremen standen die Ansätze, die das Verständnis der Möglichkeiten und der Grenzen der Methoden für unabdingbar erklärten. Dafür musste auch Basiswissen der Physik gelehrt werden. Oft wurde es fast unbewusst, sozusagen huckepack, mit den eingesetzten Methoden transportiert. So erinnerte sich Gutowsky an ein 
Gespräch mit seinem Mentor in Harvard, E. Bright Wilson, der in den 1930er Jahren gemeinsam mit Linus Pauling eine stark mathematisch und theoretisch orientierte Einführung in die Quantenchemie geschrieben hatte:

I commented on to Bright Wilson about how important in chemical education his book on quantum mechanics had been. He looked at me and laughed and he said: 'You have done more to teach quantum mechanics to chemistry students than anybody.' I said: 'I have?' He said: 'Yes. Organic chemists have had to learn second order quantum mechanics to solve the $\mathrm{AB}$ [NMR] spectrum. That taught more chemists more quantum mechanics than anyone realized. ${ }^{12}$

Für Wilson kam die physikalische Theorie tatsächlich in einem ,Paket' mit den Instrumenten und war von diesen nicht zu trennen. Diese Verbindung und die Herstellung chemischer Bezüge der physikalischen Methoden führte zu einer Verschmelzung der ursprünglich als getrennt angesehenen Bereiche der Physik und Chemie in Gestalt der neuen, physikalischen Methoden.

Aber die Grenze zwischen Physik und Chemie wurde durch das Aufkommen der neuen physikalischen Methoden in der Chemie nicht hinfällig. Indem sie diese Grenzen überwanden, ließen die von mir als Methodenmacher bezeichneten Wissenschaftler sie nicht verschwinden. Die Methoden der Physik unterscheiden sich von den physikalischen in der Chemie, und diesen Unterschied herzustellen, war das Betätigungsfeld von Wissenschaftlern wie Gutowsky, Biemann, Djerassi und vielen anderen. Verstanden sich die theoretischen Physiker als Designer theoretischer Modelle, so sahen sich die organischen Chemiker als Erbauer einer synthetischen Welt. Diese Sichten bestimmten ihren Habitus und die von ihnen vertretene soziale Hierachie: der Einzelgänger als Denker mit Bleistift und später mit Computer; der TeamWorker, der eine zupackende Mentalität an den Tag legte und dabei große Gruppen leiten musste. Dazwischen vermittelten die Methodenmacher.

\section{Habitus und Hierarchien}

Anders als bei den von Terry Shinn in der Analyse eines zeitgenössischen Physiklabors herangezogenen Auswirkungen der Hierarchien auf die Art der Forschungsstrategien (Shinn 1988), handelt es sich bei den von mir beschriebenen Hierarchien nicht nur um institutionelle, sondern vor allem um habituelle. Chemiker sahen es als selbstverständlich an, dass sie ihre Ergebnisse mit der eigenen Hände Arbeit (meist allerdings der ihrer Doktoranden) und im Experimentieren mit chemischen Substanzen erzielten. Dies hatte Auswirkungen auf die Hierarchie in den Laboratorien und erschwerte die Akzeptanz der neuen Methoden, die diese Praktiken nicht mehr zuließen und neue Abhängigkeiten schufen. Physiker dagegen sahen sich stärker als Designer genereller theoretischer Modelle, im besten Fall als Konstrukteure 
generischer Instrumente (zu letzterem Joerges/Shinn 2001). Dies führte zu einer Abwertung der Forscher, die an den Anwendungen der Methoden und der Instrumente arbeiteten. Sollte ein neues Feld zwischen Physik und Chemie geschaffen werden, so waren die Habitusformen der Forscher soweit anzugleichen, dass sie miteinander auskommen konnten. Im Fall von NMR und auch der Massenspektrometrie wurden neue eigenständige Gemeinschaften gebildet, an deren Entstehen nicht nur die Forscher selbst, sondern auch die Instrumentenhersteller und die staatliche Wissenschaftsförderung mitwirkten (Reinhardt/Steinhauser 2008).

Das hier exemplarisch angeführte Aufeinanderprallen verschiedener Habitusformen und der mit ihnen verbundenen Hierarchien ist vermutlich auch bei anderen Neuausbildungen wissenschaftlicher Gemeinschaften oder Felder zu erkennen. Die Perspektive der Methodenmacher erlaubt es, die Konfliktlinien besonders deutlich zu sehen, da die Verfügungsgewalt über die Methoden die Zugänge zu den Forschungsobjekten ebenso regelt wie die soziale Hierarchie im Feld. Es ist also zu vermuten, dass die Einführung anderer Forschungstechnologien als der NMR und der Massenspektrometrie Parallelen zu den hier geschilderten Vorkommnissen zeigt. Auch bei der Entwicklung der Materialwissenschaften dürften sich solche Phänomene beobachten lassen, sind doch auch für sie neuartige Forschungsmethoden konstitutiv. Die Situation stellt sich allerdings komplexer dar als in dem hier benutzten Beispiel, da zahlreiche verschiedenartige Disziplinen beteiligt sind - neben den Naturwissenschaften auch technische - und die Rolle der Wissenschafts- und Technikförderung stärker zu berücksichtigen wäre. Mein Beitrag versteht sich auch als methodische Anregung für weiterführende Studien in dieser Richtung.

Dabei wäre zu beachten, inwieweit der Transfer von Instrumenten und Theorien zwischen den Disziplinen oder Feldern auch den Transfer eines spezifischen Habitus mit sich brachte. Mit anderen Worten: Ob der Gebrauch eines physikalischen Instruments den Anwender in der Chemie oder einer anderen Disziplin dazu zwang, sich habituell wie ein Physiker zu verhalten. Die hier vorgestellten Beispiele legen nahe, dass dem nur eingeschränkt so war. Die Chemiker versuchten, ihre habituelle Prägung fortzuführen, die Instrumente und Methoden einzuhegen, allenfalls marginal zuzulassen. Die Physiker blieben bei ihren theoretischen und generellen Zielen. Es war dagegen eine sich neu ausbildende Gruppierung von Forschern, die die Entwicklung von Methoden zu ihrem Programm machte. In der Folge kam es zur Bildung eines neuen Feldes, das sich durch einen spezifischen Habitus auszeichnete und dadurch auch auf längere Sicht etablieren konnte. Die Frage, ob die Materialwissenschaften ein Konglomerat lose verbundener Teile oder ein eigenständiges Feld darstellen, dürfte sich demnach auch über die Beobachtung eines spezifischen Habitus beantworten lassen. 


\section{Danksagung}

Der Beitrag geht auf einen Vortrag zurück, den ich auf der gemeinsamen Tagung der Fachgruppe Geschichte der Chemie der Gesellschaft Deutscher Chemiker (GDCh) und dem Fachverband Geschichte der Physik der Deutschen Physikalischen Gesellschaft (DPG) im März 2009 in Göttingen gehalten habe. Ich danke Paula Diehl für die Anregung, Bourdieus Theorie heranzuziehen; Klaus Hentschel und Christoph Meinel sowie drei anonymen Gutachterinnen und Gutachtern für kritische Hinweise.

\section{Anmerkungen}

1 Norman Ramsey an Herbert Gutowsky, 25. Oktober 1950, Gutowsky Nachlass, in Privatbesitz.

2 Gutowsky an Ramsey, 27. November 1950, ebd. Ausführliche Zitate aus dem Briefwechsel und eine vertiefte Darstellung der Schwierigkeiten der Zusammenarbeit in Reinhardt 2006a: 55-61, hier $59 \mathrm{f}$.

3 Dies geschieht in kursorischer, resümeeartiger Weise auf der Basis meiner Arbeiten zum Thema, vor allem Reinhardt 2006a.

4 Siehe Abderhalden 1912, Weissberger 1945-1946 und zu Houben/Weyl: Müller 1955. Für die physikalische Chemie ist Reilly/Rae 1926 ein frühes Beispiel. Die Subdisziplin Anorganische Chemie folgte erst 1963, mit Technique of Inorganic Chemistry erschien auch ein nur unspezifischer Titel.

5 Die folgenden Zitate sind bis auf eine Ausnahme meinen Arbeiten zum Thema entnommen, vor allem Reinhardt 2004, 2006a. Sie sind dort jeweils analysiert und in die Forschungen der Akteure eingebettet, aber nicht zusammenhängend unter dem in diesen Aufsatz propagierten Ansatz untersucht worden.

6 Das Zitat stammt aus einem Interview, das ich am 1. und 2. Dezember 1998 mit Herbert Gutowsky führte. Es ist abgedruckt in Reinhardt 2004: 28, dort findet sich eine detaillierte Einordnung in den Forschungskontext Gutowskys und Pakes.

7 Es handelte sich hier um die sogenannte Spin-Spin-Kopplung, einen der Basiseffekte für die chemische Anwendung der Methode.

8 Autrey an Biemann, 9. Oktober 1962, Biemann Nachlass, in Privatbesitz. Dieses Zitat und die Antwort sind abgedruckt in Reinhardt 2006a: 125, dort auf S. 123-126 auch eine detaillierte Analyse der Auseinandersetzung. Siehe auch Reinhardt 2002.

9 Biemann an Autrey, 18. Oktober 1962, Biemann Nachlass, in Privatbesitz.

10 Gutowsky im Interview mit dem Autor, 1. und 2. Dezember 1998. Vgl. auch Reinhardt 2006a: 34-35, 383. Zu einer allgemeinen Schilderung der Servicelabors in Departments sowie auf regionaler und nationaler Ebene siehe Reinhardt 2006b.

11 Zu Djerassis Analogie siehe Reinhardt 2006a: 369-370 und eine seiner Autobiographien, Djerassi 1992: 84. Das folgende Zitat ist aus einem Interview des Autors mit Djerassi vom 20. Januar 1999, abgedruckt in Reinhardt 2006a: 169-170.

12 Interview mit dem Autor, 1. und 2. Dezember 1998, Zitat abgedruckt in Reinhardt 2006a: 359.

\section{Literatur}

Abderhalden, Emil, 1912. Physiologisches Praktikum. Chemische und Physikalische Methoden. Berlin: Springer. 
Bensaude-Vincent, Bernadette, 2001. The Construction of a Discipline: Materials Science in the United States. Historical Studies in the Physical Sciences, 31, 223-248.

Bourdieu, Pierre, 1993 [1980]. Sozialer Sinn. Kritik der theoretischen Vernunft. Frankfurt a. M.: Suhrkamp.

Bourdieu, Pierre, 1998. Vom Gebrauch der Wissenschaft. Für eine klinische Soziologie des wissenschaftlichen Feldes. Konstanz: UVK Universitätsverlag.

Bourdieu, Pierre, 1999 [1979]. Die feinen Unterschiede. Kritik der gesellschaftlichen Urteilskraft. Frankfurt a. M.: Suhrkamp.

Diehl, Paula, 2005. Macht - Mythos - Utopie. Die Körperbilder der SS-Männer. Berlin: AkademieVerlag.

Djerassi, Carl, 1992. The Pill, Pygmy Chimps, and Degas' Horse. The Autobiography of Carl Djerassi. New York: Basic Books.

Fleck, Ludwik, 1980 [1935]. Entstehung und Entwicklung einer wissenschaftlichen Tatsache. Einführung in die Lehre vom Denkstil und Denkkollektiv. Frankfurt a. M.: Suhrkamp.

Heering, Peter, 2007. Das Konzept des Experimentierstils zur Beschreibung historischer Experimentalpraxis. In: Bozena Choluj und Jan C. Joerden, Hg., Von der wissenschaftlichen Tatsache zur Wissensproduktion: Ludwik Fleck und seine Bedeutung für die Wissenschaft und Praxis. Frankfurt a. M.: Peter Lang, 361-385.

Hentschel, Klaus, 2010. Von der Werkstoffforschung zur Materials Science, NTM. Zeitschrift für Geschichte der Wissenschaften, Technik und Medizin, 18(4), 1-36.

Joerges, Bernward/Shinn, Terry, Hg., 2001. Instrumentation between Science, State and Industry. Dordrecht: Kluwer Academic Publishers.

Johnson, Jeffrey A., 1989. Hierarchy and Creativity in Chemistry 1871-1914. Osiris, 5, 214-240.

Jordan, Kathleen/Lynch, Michael, 1998. The Dissemination, Standardization and Routinization of a Molecular Biology Technique, Social Studies of Science, 28, 773-800.

Knoblauch, Hubert, 2005. Wissenssoziologie. Konstanz: UVK.

Laszlo, Pierre, 2002. Tools, Instruments and Concepts: The Influence of the Second Chemical Revolution. In: Peter J. T. Morris, Hg., From Classical to Modern Chemistry. Cambridge: Royal Society of Chemistry, 171-187.

Lynch, Michael/Jasanoff, Sheila, 1998. Introduction: Contested Identities. Science, Law and Forensic Practice. Social Studies of Science, 28, 675-686.

Morgan, Mary/Morrison, Margaret, Hg., 1999. Models as Mediators: Perspectives on Natural and Social Science. Cambridge: Cambridge University Press.

Müller, Eugen, Hg., 1955. Physikalische Forschungsmethoden. Bd. 3. Methoden der Organischen Chemie. Stuttgart: Thieme.

Niethammer, Lutz, 2000. Kollektive Identität. Heimliche Quellen einer unheimlichen Konjunktur. Hamburg: Rowohlt.

Nye, Mary Jo, 1993. From Chemical Philosophy to Theoretical Chemistry. Dynamics of Matter and Dynamics of Disciplines, 1800-1950. Berkeley: University of California Press.

Park, Buhm Soon, 2003. The ,Hyperbola of Quantum Chemistry'. The Changing Practice and Identity of a Scientific Discipline in the Early Years of Electronic Digital Computers, 1945-65. Annals of Science, 60, 219-247.

Perrin, Carleton E., 1988. Research Traditions, Lavoisier, and the Chemical Revolution. Osiris, 4, 53-81.

Rabkin, Yakov M., 1987. Technological Innovation in Science. The Adoption of Infrared Spectroscopy by Chemists. Isis, 78, 31-54.

Reilly, Joseph/Rae, William Norman, 1926. Physico-Chemical Methods. London: Methuen \& Co.

Reinhardt, Carsten, 2002. The Chemistry of an Instrument. Mass Spectrometry and Structural Organic Chemistry. In: Peter J. T. Morris, Hg., From Classical to Modern Chemistry. Cambridge: Royal Society of Chemistry, 229-247.

Reinhardt, Carsten, 2004. Chemistry in a Physical Mode. Molecular Spectroscopy and the Emergence of NMR. Annals of Science, 61, 1-32.

Reinhardt, Carsten, 2006a. Shifting and Rearranging. Physical Methods and the Transformation of Modern Chemistry. Sagamore Beach, MA: Science History Publications.

Reinhardt, Carsten, 2006b. Wissenstransfer durch Zentrenbildung. Physikalische Methoden in der Chemie und den Biowissenschaften. Berichte zur Wissenschaftsgeschichte, 29, 224-242.

Reinhardt, Carsten, 2007. Physikalische Methoden in der Chemie, ca. 1960. Implementierung und Strategien der Durchsetzung, Dahlemer Archivgespräche, 13, 29-48. 
Reinhardt, Carsten/Steinhauser, Thomas, 2008. Formierung einer wissenschaftlich-technischen Gemeinschaft. NMR-Spektroskopie in der Bundesrepublik Deutschland. NTM. Zeitschrift für Geschichte der Wissenschaften, Technik und Medizin, 16, 73-101.

Schummer, Joachim, 2002. The Impact of Instrumentation on Chemical Species Identity. In: Peter J. T. Morris, Hg., From Classical to Modern Chemistry. Cambridge: Royal Society of Chemistry, 188-211.

Shinn, Terry, 1988. Hiérarchies des chercheurs et formes des recherches. Actes de la recherche en sciences sociales, 74, 2-22.

Slater, Leo, 2002. Organic Chemistry and Instrumentation: R. B. Woodward and the Reification of Chemical Structures. In: Peter J. T. Morris, Hg., From Classical to Modern Chemistry. Cambridge: Royal Society of Chemistry, 212-228.

Smith, Laurence D./Best, Lisa A./Stubbs, D. Alan/Johnston, John/Archibald, Andrea Bastiani, 2000. Scientific Graphs and the Hierarchy of the Sciences: A Latourian Survey of Inscription Practices. Social Studies of Science, 30, 73-94.

Steinhauser, Thomas, 2009. Zukunftsmaschinen in der Chemie. Kernmagnetische Resonanz bis 1980. Unveröffentlichte Dissertation, Universität Regensburg.

Straub, Jürgen, 1998. Personale und kollektive Identität. Zur Analyse eines theoretischen Begriffs. In: Assmann, Aleida und Heidrun Friese, Hg., Identitäten. Frankfurt am Main: Suhrkamp, 73104 [Erinnerung, Geschichte, Identität, 3].

Treibs, Alfred, 1971. Das Leben und Werk von Hans Fischer. München: Hans Fischer-Gesellschaft. Weissberger, Arnold, 1945-46. Physical Methods of Organic Chemistry. 2 Bde. New York City: Interscience.

\author{
Carsten Reinhardt \\ Institut für Wissenschafts- und Technikforschung \\ Universität Bielefeld \\ Postfach 100131 \\ 33501 Bielefeld \\ Deutschland \\ E-Mail: carsten.reinhardt@uni-bielefeld.de
}

